\title{
Gender effects on odor-stimulated functional magnetic resonance imaging
}

\author{
David M. Yousem ${ }^{\text {a,b, },}$, Joseph A. Maldjian ${ }^{\text {a }}$, Faez Siddiqi a, Thomas Hummel ${ }^{\text {b }}$, David C. Alsop ${ }^{\text {a }}$, \\ Rena J. Geckle ${ }^{\mathrm{a}, \mathrm{b}}$, Warren B. Bilker ${ }^{\mathrm{c}}$, Richard L. Doty ${ }^{\mathrm{b}}$ \\ ${ }^{a}$ Department of Radiology, University of Pennsylvania Medical Center, 3400 Spruce Street, Philadelphia, PA 19104, USA \\ ${ }^{\mathrm{b}}$ University of Pennsylvania Smell and Taste Center, University of Pennsylvania Medical Center, 3400 Spruce Street, Philadelphia, PA 19104, USA \\ ${ }^{\mathrm{c}}$ Department of Biostatistics and Epidemiology and Center for Clinical Epidemiology and Biostatistics, University of Pennsylvania Medical Center, 3400 \\ Spruce Street, Philadelphia, PA 19104, USA
}

\begin{abstract}
On standardized tests of odor identification and odor detection, women tend to score better than men at nearly all age groups. We sought to determine if these findings would translate to differences between the sexes in the volume of activated brain when odors are presented to subjects as the stimulants for functional magnetic resonance imaging (FMRI) experiments. The activation maps of eight right-handed women (mean age 25.3 years old, range 20-44, S.D. 8.3 years) were compared with those of 8 right-handed men (mean age 30.5, range 18-37, S.D. 6.5 years) given the same olfactory nerve stimuli in an FMRI experiment at 1.5 T. Olfactory stimuli were delivered to the patients in a passive fashion using a Burghart OM4-B olfactometer with a nose piece inserted into the patients' nostrils. We used agents (eugenol, phenyl ethyl alcohol, or phenyl ethyl alcohol alternating with hydrogen sulfide) that were selective for olfactory nerve stimulation in the nose. The odorants were delivered to both nostrils for $1 \mathrm{~s}$ every $4 \mathrm{~s}$ during a $30 \mathrm{~s}$ 'on-period'. During the $30 \mathrm{~s}$ 'off-period', the patient received room air at the same flow rate. The women's group-averaged activation maps showed up to eight times more activated voxels than men for specific regions of the brain (frontal and perisylvian regions). The left and right inferior frontal regions showed a statistically significant increase in activation in women at $p<0.01$. In general, more women showed activation than men. The results suggest that (1) FMRI activation maps in subject groups can demonstrate correlates to psychophysical tests of olfaction, and (2) one must control for gender when performing odor-stimulated FMRI experiments. (C) 1999 Elsevier Science B.V. All rights reserved.
\end{abstract}

Keywords: Olfaction; Smell; Odor; Functional magnetic resonance imaging; Brain mapping; Gender effect; Cortex

\section{Introduction}

Despite the fact that the sense of smell is common to nearly all creatures in the animal kingdom and is centered in a portion of the brain that is thought to develop early in phylogeny, the understanding of odor processing and the mechanisms controlling integration of odor-associated memories and emotions is quite limited. Only a few positron emission tomography (PET) studies [36,37] and a few preliminary functional magnetic resonance imaging (FMRI) studies [27,33-35] have been published using smell stimulation. These studies have shown either an increase in regional blood flow (PET) or activation of cerebral voxels (FMRI) in the orbitofrontal regions (right more than left) with odor stimulation.

\footnotetext{
* Corresponding author. Fax: + 1-215-662-3283; E-mail: yousem@oasis.rad.upenn.edu
}

We sought to analyze the effects of gender on cortical activation with odor-stimulated FMRI (OSFMRI) studies. Doty et al. have shown that women tend to perform better than men across all age groups in tests of odor identification and odor detection thresholds [5,7-9]. We hypothesized that, compared to men, women would show an increase in the number of activated voxels with OSFMRI.

\section{Materials and methods}

\subsection{Subject selection}

All subjects were initially screened for any possible causes of smell dysfunction prior to being invited to enter the study. The subjects completed a standardized handedness survey [4] to insure that they were all right-handed. All subjects also underwent odor identification testing with the 40 item University of Pennsylvania Smell Identifica- 
tion Test (UPSIT) (20 items each nostril) to ensure that their sense of smell was appropriate for age [5-7]. The project was reviewed and approved by our hospital's institutional review board and patients completed informed consent forms.

Eight right-handed women (mean age 25.3 years old, range 20-44, S.D. 8.3 years) were compared with eight right-handed men (mean age 30.5, range 18-37, S.D. 6.5 years) to examine gender effects. The scores of the women on the 40 item UPSIT ranged from 33-39 with a mean of 38.5 and S.D. of 1.1 and the men's values ranged from 31-40, mean 35.7, and S.D. of 2.7. As groups, these values fall within expected norms for age and gender [13].

\subsection{FMRI protocol}

The studies were performed on a 1.5 T GE Horizon echospeed scanner. Localization for the FMRI studies consisted of a T1-weighted (T1W) sagittal scan with repetition time $\left(T_{\mathrm{R}}\right)$ of $500 \mathrm{msec}$, time to echo $\left(T_{\mathrm{E}}\right) 11 \mathrm{msec}$, and 1 average $(500 / 11 / 1)$. This scan was followed by an axial $500 / 11 / 1$ scan with a $192 \times 256$ matrix and $5 \mathrm{~mm}$ thick interleaved sections through the entire brain. These conventional T1-weighted axial images were used for performing anatomic overlays. An echoplanar chemical shift imaging sequence was also performed to measure the magnetic field within the brain of the subject. This information was used to correct for spatial shifts and distortion in the echoplanar images that can cause misalignment with the T1-weighted anatomic images.

Functional MRI studies were performed in the axial plane using multislice gradient echo echoplanar imaging. Scan parameters included a $64 \times 40$ matrix, $24 \times 15 \mathrm{~cm}^{2}$ field of view, $T_{\mathrm{R}}$ of $3000 \mathrm{msec}, T_{\mathrm{E}}$ of $30 \mathrm{msec}, 5 \mathrm{~mm}$ thickness, and a $90^{\circ}$ flip angle, delivering a voxel resolution of approximately $4 \times 4 \times 5 \mathrm{~mm}^{3}$. A total of 120 images were acquired at each of 24 slice locations per paradigm over the course of a 6 min FMRI scan. Each task paradigm consisted of 10 alternating rest-stimulus cycles (30 s each) over the $6 \mathrm{~min}$.

\subsection{Olfactory stimulant delivery}

Olfactory stimuli were delivered to the patient using a Burghart OM4-B olfactometer (Wedel, Germany) with a continuous flow method $(4 \mathrm{l} / \mathrm{min})$. This machine delivers the stimulants through tubing terminating in a nose piece inserted into the patients' nostrils. The nose piece is similar in size to oxygen nasal cannula tubing; however, the air flow and humidity can be precisely regulated by the olfactometer [26]. Odors were delivered to both nostrils for $1 \mathrm{~s}$ every $4 \mathrm{~s}$ during the $30 \mathrm{~s}$ 'on-period'. During the $30 \mathrm{~s}$ 'off-period', the patient received room air at the same flow rate.

Before and after each scan, the subject completed a questionnaire as to the perceived quality and intensity of the stimulants used. This step helped to ensure that odor delivery had occurred in a consistent, reliable manner.

To study the effects of gender we used agents (eugenol, phenyl ethyl alcohol, or phenyl ethyl alcohol alternating with hydrogen sulfide) that are relatively selective for olfactory nerve stimulation in the nose. These odorants are rarely detected by anosmics (who cannot detect olfactory nerve stimulants but can detect trigeminal nerve stimulants) or by subjects trained to detect trigeminal stimulation in the nose $[9,25]$. In total there were 10 paradigms employing the phenyl ethyl alcohol-hydrogen sulfide combination, three paradigms employing just phenyl ethyl alcohol, and two paradigms employing eugenol alone (total $=15$ ) performed in the women. The same number and type of paradigms were performed in the men. The paradigms were repeated in five women and six men for studies of reproducibility.

\subsection{Data analysis}

The FMRI raw echo amplitudes were saved and transferred to a SUN Ultrasparc 1 (SUN Microsystems, Mountain View, CA) for off-line reconstruction using in-house software developed in IDL (Research Systems, Boulder, $\mathrm{CO}$ ). Correction for image distortion and alternate k-space line errors was performed on each image on the basis of data acquired during phase-encoded reference imaging. Statistical parametric maps (SPMs) were generated using SPM96 [16,17,19,20] (from the Wellcome Dept. of Cognitive Neurology, London, UK) implemented in Matlab (Mathworks, Sherborn, MA, USA), with an IDL (Research Systems, Boulder, CO) interface. The IDL interface was developed in-house in collaboration with the UMDNJ FMRI lab (Newark, NJ). The T1-weighted images were normalized to a standard atlas (Talairach space). The functional data sets were motion corrected (intra-run realignment) within SPM96 using first- and second-order motion correction, as well as a first-order correction for spin history, using the first image as the reference [15,18]. The functional data sets were normalized to Talairach space using image header information to determine the 16parameter affine transform between the functional data sets and the T1-weighted images in combination with the transform computed within SPM96 for the T1-weighted anatomic images to Talairach space. The normalized data sets were resampled to $4 \times 4 \times 5 \mathrm{~mm}^{3}$ within Talairach space using sinc interpolation. A second realignment step (inter-run realignment) was then performed (within SPM96) between successive normalized runs within each subject, using the initial normalized run as the reference. This was done to eliminate motion between the successive runs within each subject. The data sets were smoothed using a $8 \times 8 \times 10 \mathrm{~mm}^{3}$ full-width at half-maximum Gaussian smoothing kernel, and SPMs were generated using the general linear model within SPM96 [16,18]. A 6 s timeshifted box-car waveform was used as the reference 
Table 1

Mann-Whitney test results comparing number of voxels activated in eight men and eight women by site when threshold for activation was set at $p<0.01$

\begin{tabular}{lllllllllll}
\hline & RIF & LIF & RIMT & LIMT & RPS & LPS & RC & LC & RSF & LSF \\
\hline Mann-Whitney $p$-values & $0.0297^{*}$ & $0.0328^{*}$ & 0.111 & 0.171 & 0.792 & 0.737 & 0.267 & 0.180 & 0.240 & 0.205 \\
\hline
\end{tabular}

${ }^{*} p<0.05$.

$\mathrm{RIF}=$ right inferior frontal, LIF $=$ left inferior frontal, RIMT $=$ right inferomedial temporal lobe, LIMT $=$ left inferomedial temporal lobe, $\mathrm{RPS}=$ right perisylvian, $\mathrm{LPS}=$ left perisylvian, $\mathrm{RC}=$ right cingulate, $\mathrm{LC}=$ left cingulate, $\mathrm{RSF}=$ right superior frontal, $\mathrm{LSF}=$ left superior frontal.

paradigm, and the ANCOVA model with global activity as a confound was employed for the statistical analysis. In addition, high pass and low pass filters were employed as confounds within the analysis. The resulting set of images represent statistical parametric maps of the $t$ statistic $\operatorname{SPM}\{t\}$. Individual SPMs were generated for every run for each subject. The SPM $\{t\}$ were transformed to the unit normal distribution $\operatorname{SPM}\{Z\}$. Group SPMs were also constructed across subjects for each condition using the smoothed normalized data sets. The thresholded SPMs were overlaid onto the anatomic normalized images for display within IDL. Temporal smoothing and detrending were performed as part of the SPM analysis.

Volumetric analysis was performed at a threshold of $p=0.05$ for each group-averaged map and the number of voxels activated was computed for the right and left frontal and temporal lobes. The superior and posterior margins of the Sylvian fissures were used as the boundaries for the temporal lobe. The central sulcus posteriorly and Sylvian fissure inferiorly were used to delineate the frontal lobe. To determine whether the ratios of activation were dependent on the threshold set, the group maps were also evaluated at $p$-values of $0.025,0.015$, and 0.01 .

For the volumetric analysis for individual maps (Tables 4 and 1) the number of voxels activated at a $p$-value of 0.01 was computed for the right and left inferior and superior frontal lobes, the right and left perisylvian regions, the right and left inferomedial temporal lobes, and the right and left cingulate gyri. These regions were defined using standard neuroanatomic landmarks. The superior and posterior margins of the Sylvian fissures were used as the boundaries for the temporal lobe, and the central sulcus posteriorly and Sylvian fissure inferiorly were used to delineate the frontal lobe. The inferior frontal lobe was defined as that portion of the frontal lobe below the genu of the corpus callosum in the Talairach maps. The inferior frontal lobe included the orbitofrontal, frontopolar and gyrus rectus regions. The perisylvian region included the insula and the regions of the temporal lobe bounded by the sylvian fissure. The inferomedial temporal lobe was defined as that region that was located inferior and medial to the temporal horns of the lateral ventricles. This region included the entorhinal and piriform cortices, as well as the hippocampus and parahippocampus. Comparison of the activations levels observed for male and females was made in each of these 10 measured regions using the MannWhitney test (Table 1). The Mann-Whitney test was used, as opposed to the $t$-test, due to the observed non-normality of the activations.

To assess whether the ratios of activation were dependent on the thresholds chosen, each individual map was assessed at $p$-values of $0.02,0.01$, and 0.005 . The number of activated voxels above these thresholds were recorded for each of the 10 sites listed above and then subjected to the Mann-Whitney test.

\section{Results}

\subsection{Volume of activation}

The activation in both male and female subjects was greater in the right frontal region than the left frontal region by a factor of over 4 ( 730 voxels vs. 157 voxels) for women and 8 (152 voxels vs. 19 voxels) for men when the group maps were thresholded at a $p$-value of 0.05 (Table 2 ). The temporal lobe activation was slightly greater on the right for women (ratio 1.5/1) and on the left for men

Table 2

Analysis of activation using group-averaged maps between men and women thresholded at a $p$-value of 0.05

\begin{tabular}{lllll}
\hline $\begin{array}{l}\text { Subject } \\
\text { group }\end{array}$ & $\begin{array}{l}\text { No. of left frontal } \\
\text { voxels activated }\end{array}$ & $\begin{array}{l}\text { No. of right frontal } \\
\text { voxels activated }\end{array}$ & $\begin{array}{l}\text { No. of left temporal } \\
\text { voxels activated }\end{array}$ & $\begin{array}{l}\text { No. of right temporal } \\
\text { voxels activated }\end{array}$ \\
\hline $\begin{array}{l}\text { Female, } \\
\text { right-handed, } \\
<50 \text { years old }\end{array}$ & 157 & 730 & 303 & 465 \\
$\begin{array}{l}\text { Male, } \\
\text { right-handed, } \\
<50 \text { years old }\end{array}$ & 19 & 152 & 55 & 31 \\
\hline
\end{tabular}

Note that in all sites, women showed more activated voxels than men. The difference was most striking in the right temporal (peri-insular) regions. 

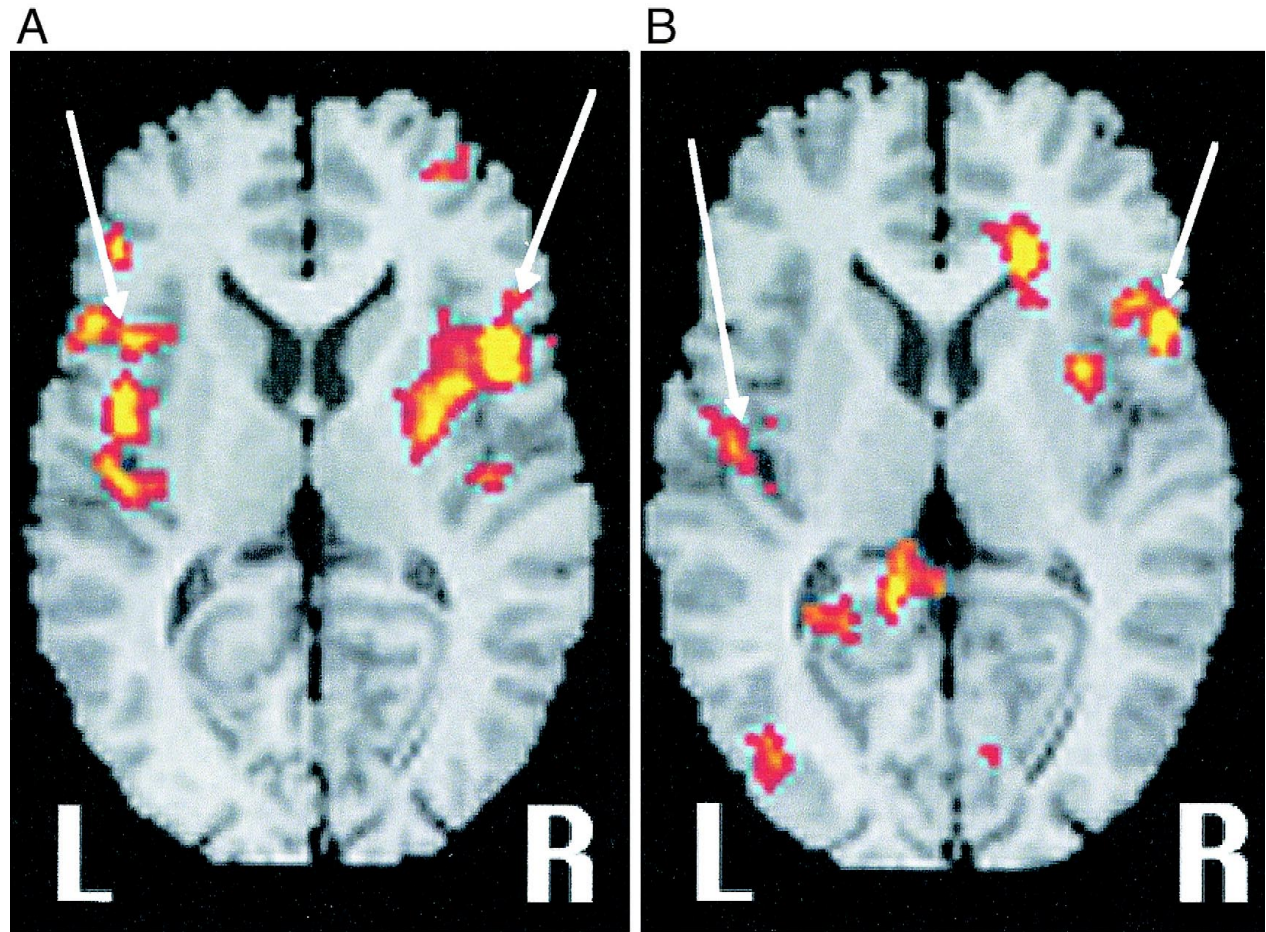

Fig. 1
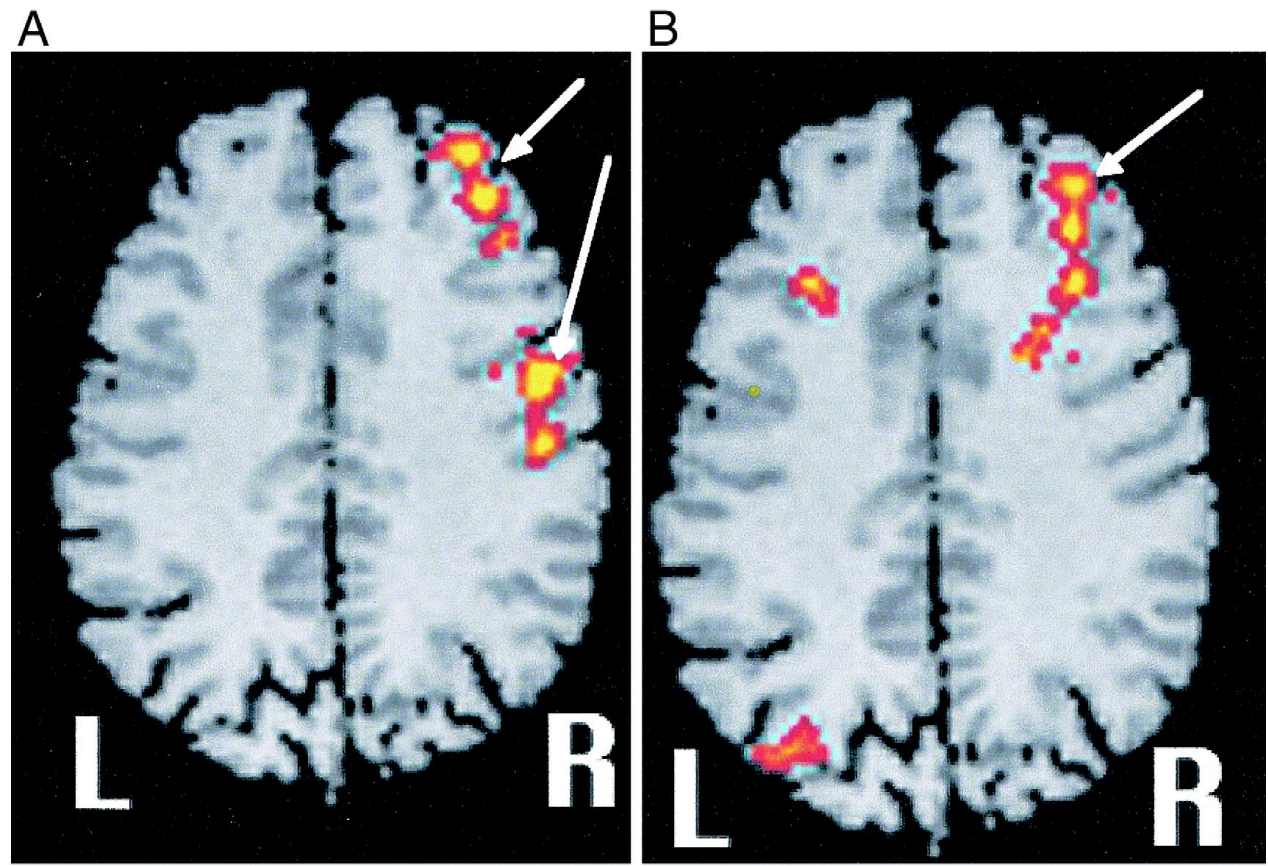

Fig. 2

Fig. 1. Differences demonstrated in one selected image through the peri-insular regions. (A) Both peri-insular regions (arrows) show a large number of activated voxels in the group-averaged map of the women. (B) The degree of activation in the right and left peri-insular zones (arrows) is much reduced in the men.

Fig. 2. Right frontal activation. Both the women (A) and the men (B) evidence greater activation in the right frontal lobe (arrow) than the left. Overall, the women showed more than a four-fold greater number of activated voxels on the right. In other sections, the women also showed far greater left frontal activation. 
Table 3

Ratios of activation based on Table 2

\begin{tabular}{lllllll}
\hline Variable & All sites & $\begin{array}{l}\text { Left and } \\
\text { right frontal }\end{array}$ & $\begin{array}{l}\text { Left and } \\
\text { right temporal }\end{array}$ & $\begin{array}{l}\text { Left } \\
\text { frontal }\end{array}$ & $\begin{array}{l}\text { Right } \\
\text { frontal }\end{array}$ & $\begin{array}{l}\text { Left } \\
\text { temporal }\end{array}$ \\
\hline $\begin{array}{l}\text { Female to male, } \\
\text { right-handed, } \\
<50 \text { years old }\end{array}$ & 6.4 & 5.2 & 8.9 & 8.3 & 4.8 & 5.5 \\
temporal
\end{tabular}

By dividing the number of activated voxels in women by those in men, one can observe the marked differences in the temporal lobes and left frontal regions.

$(1.8 / 1)$. Both perisylvian regions showed the greatest degree of temporal lobe activation (Fig. 1). The total number of voxels activated in the brain was 6.4 times greater for women than men, with the differences most prominent in the right temporal lobe and left frontal lobe (Table 3). The frontal regions that were activated corresponded to Brodmann areas 6,10 , and 46 . The activated perisylvian cortex corresponded to Brodmann areas 6 and 22.

When these group maps were thresholded at $p$-values of 0.025 , the right-sided predilection for activation persisted in men and women by a factor of 2.6 to 1 for the frontal lobe and 2 to 1 in the temporal lobe. At a $p$-value of 0.015 , there were 4.4 times more activated voxels in the right frontal lobe than left and 1.9 times more voxels

Table 4

Number of voxels activated in each region by subject ( $p$-value threshold set at 0.01 )

\begin{tabular}{lcccrrrrrrr}
\hline Subject & RIF & LIF & RIMT & LIMT & RPS & LPS & RC & LC & RSF & LSF \\
\hline Male 1 & 1 & 4 & 1 & 0 & 10 & 12 & 5 & 0 & 25 & 0 \\
Male 2 & 0 & 1 & 0 & 0 & 0 & 0 & 0 & 0 & 1 & 1 \\
Male 3 & 0 & 0 & 7 & 0 & 19 & 14 & 0 & 0 & 11 & 0 \\
Male 4 & 0 & 0 & 0 & 0 & 0 & 0 & 0 & 0 & 0 & 0 \\
Male 5 & 0 & 0 & 0 & 0 & 7 & 0 & 0 & 0 & 1 & 0 \\
Male 6 & 0 & 0 & 16 & 46 & 2 & 18 & 0 & 0 & 0 & 0 \\
Male 7 & 0 & 0 & 5 & 6 & 14 & 0 & 0 & 0 & 1 & 0 \\
Male 8 & 0 & 0 & 0 & 0 & 10 & 0 & 0 & 0 & 4 & 3 \\
Female 1 & 2 & 7 & 0 & 0 & 1 & 0 & 4 & 0 & 3 & 0 \\
Female 2 & 6 & 3 & 0 & 0 & 42 & 21 & 0 & 1 & 1 & 0 \\
Female 3 & 0 & 9 & 0 & 0 & 11 & 7 & 52 & 45 & 3 & 0 \\
Female 4 & 0 & 0 & 0 & 0 & 6 & 0 & 0 & 0 & 0 & 0 \\
Female 5 & 2 & 7 & 4 & 0 & 42 & 5 & 11 & 0 & 63 & 2 \\
Female 6 & 23 & 0 & 0 & 0 & 5 & 0 & 0 & 0 & 10 & 5 \\
Female 7 & 16 & 2 & 0 & 0 & 2 & 6 & 0 & 4 & 1 & 34 \\
Female 8 & 0 & 4 & 0 & 0 & 2 & 2 & 0 & 4 & 11 & 11 \\
All men & $1^{\mathrm{a}}$ & $5^{\mathrm{a}}$ & 29 & 52 & 61 & 34 & 5 & 16 & 43 & 4 \\
added & & & & & & & & & & \\
together & & & & & & & & & & \\
All & $49^{\mathrm{a}}$ & $32^{\mathrm{a}}$ & 4 & 0 & 109 & 41 & 67 & 46 & 100 & 52 \\
women added & & & & & & & & & \\
together & & & & & & & & & \\
\hline
\end{tabular}

$\mathrm{RIF}=$ right inferior frontal, $\mathrm{LIF}=$ left inferior frontal, $\mathrm{RIMT}=$ right inferomedial temporal lobe, LIMT $=$ left inferomedial temporal lobe, $\mathrm{RPS}=$ right perisylvian, $\mathrm{LPS}=$ left perisylvian, $\mathrm{RC}=$ right cingulate, $\mathrm{LC}=$ left cingulate, $\mathrm{RSF}=$ right superior frontal, $\mathrm{LSF}=$ left superior frontal.

${ }^{a}$ Significant differences at Mann-Whitney test $p<0.05$. activated in the right temporal lobe than the left. No voxels survived a threshold of $p=0.01$ in the group maps.

\subsection{Incidence of activation}

When individual subjects were studied, we found that all female subjects and seven out of eight male subjects showed some degree of activation, but the site of that activation varied widely. Six of eight men activated the right superior frontal region and the right perisylvian region (Fig. 2). Half of the men activated the left perisylvian and right inferomedial temporal zone. All other sites showed activated voxels in less than half of the men (Table 5).

Individual activation maps in women demonstrated activated voxels in the right superior frontal region and right perisylvian region in all eight women. The other regions in which more than half the women showed activated voxels were the left perisylvian region, the left inferior frontal lobe and the right inferior frontal lobe (Table 5).

When the Mann-Whitney test was performed to assess for differences between the sexes based on the volumes of activation in the individual maps when the thresholds were set at $p<0.01$, women showed significantly more activation in the right and left inferior frontal lobes than men. The increased activation in women over men remained statistically significant at $p$-values thresholded $0.005,0.01$, and 0.02 , but the difference in right inferior frontal activation dropped out when the $p$-value for thresholding was set at 0.005 . No other differences between the genders was noted. Overall activation was greater in women at all

Table 5

Number of subjects who showed activated voxels in various regions

\begin{tabular}{lllllllllll}
\hline Subjects & RIF & LIF & RIMT & LIMT & RPS & LPS & RC & LC & RSF & LSF \\
\hline $\begin{array}{l}\text { Males } \\
(n=8)\end{array}$ & 1 & 2 & 4 & 2 & 6 & 3 & 1 & 1 & 6 & 2 \\
$\begin{array}{l}\text { Females } \\
(n=8)\end{array}$ & 5 & 6 & 1 & 0 & 8 & 5 & 3 & 2 & 8 & 4 \\
$\begin{array}{l}\text { Total } \\
(n=16)\end{array}$ & 6 & 8 & 5 & 2 & 14 & 8 & 4 & 3 & 14 & 6 \\
\hline
\end{tabular}

$\mathrm{RIF}=$ right inferior frontal, $\mathrm{LIF}=$ left inferior frontal, $\mathrm{RIMT}=$ right inferomedial temporal lobe, LIMT $=$ left inferomedial temporal lobe, $\mathrm{RPS}=$ right perisylvian, $\mathrm{LPS}=$ left perisylvian, $\mathrm{RC}=$ right cingulate, $\mathrm{LC}=$ left cingulate, $\mathrm{RSF}=$ right superior frontal, $\mathrm{LSF}=$ left superior frontal. 
$p$-values but the Mann-Whitney test scores showed significance only at 0.07 .

\section{Discussion}

\subsection{Location of activation}

Our results corroborate earlier FMRI findings that the right frontal lobe is intimately associated with olfaction [27,35]. On group maps of the men and women, the right frontal lobe showed more activated voxels than the left in ratios from 2.6-8.0 to 1 . In the right superior frontal lobes, the right side showed more activated voxels than the left in 11 of the 14 subjects who showed activation in this region (Table 4). These findings at FMRI corroborate the PET literature in which right frontal dominance as measured by cerebral blood flow with the $\mathrm{H}_{2} \mathrm{O}^{15}$ technique has also been demonstrated during olfactory stimulation [37]. Zatorre et al. have also demonstrated bilateral temporal lobe blood flow increases with olfactory stimulation, particularly in the peri-insula and piriform cortex regions [37].

In a previous publication [35], the site of activation was more medial and inferior to that seen currently on our study and in the recent paper of Sobel et al. [33]. We believe that this may be due to distortions in localization occurring due to susceptibility-related signal loss and different olfactory stimulation parameters. Head motion due to sniffing when odors are delivered through an oxygen mask [35] may also distort activation positioning. In any case, the sites of activation between the women and men were consistent in our study; the degree of activation was the main differentiating feature.

The bilateral nature of the temporal lobe activation was demonstrated also in this FMRI study. The right-left difference in temporal lobe activation was less striking (ratios less than 2.0) and less consistent than with the frontal lobes. The right perisylvian region demonstrated more or the same number of activated voxels in the left perisylvian zone in seven of eight women, and four of the six men who showed activation in this region. That the perisylvian region would be activated in these paradigms is not surprising since the insula has been associated with taste sensation (among other functions). Sobel et al. have also shown FMRI activation in these regions with olfactory stimulants [33].

\subsection{Gender effects on olfaction}

Gender and age are known to have strong influences on the results of psychophysical tests of olfaction [5,7-9]. One sees a progressive decline in odor identification ability in those subjects 60 years of age and older. As a group, women perform better than men on the UPSIT and on odor threshold tests at all age deciles [7,21]. Women also have larger olfactory evoked potential amplitudes than men [14] and discriminate between odors more accurately and with greater assurance than men [21]. The FMRI correlate is a diminution in numbers of voxels activated in men compared with women.

On group maps at $p$-values set at 0.05 and 0.025 and individual maps set at 0.01 , we found that women activate more voxels in the frontal and temporal lobes of their brain than men when presented with the same odorants (Tables 2 and 4$)$. There was a statistically significant $(p<0.05)$ increase in right and left inferior frontal lobe activation in women over men when the individual analysis was performed using thresholds of $p<0.01$ for activation and the left-sided inferior frontal difference was significant at activation thresholds with $p$-values of $0.005,0.01$, and 0.02 . Women showed a greater volume of activation in toto than men in all sites except the inferomedial temporal lobes (Table 4) and a greater percentage of women showed activation in the frontal lobes and temporal lobes $(100 \%$ for both) than men (75\% for both) (Table 5).

Regional metabolism of the brain as measured by PET has been reported to differ between men and women at rest [22] and during cognitive activity [22,23]. Men appear to have greater resting glucose utilization in the temporal lobes (hippocampus, amygdala), occipital-temporal regions, basal ganglia, brainstem and cerebellum, whereas women have greater glucose utilization in the cingulate regions. Cerebral blood flow [23] and cerebral metabolic rate of glucose utilization [1,3] as measured by PET tends to be increased globally in women compared to men, though the difference may not be statistically significant [1]. Contradictory findings have been reported. For example, Hatazawa et al. and Miura et al. [24,30] identified no sex differences in total brain glucose consumption or resting regional or global cerebral metabolic rate of glucose utilization, though there was some variance in glucose metabolism per unit volume. When the orbitofrontal regions were specifically tested, Andreason et al. [1] found greater rates of glucose metabolism in women than men during an auditory version of the continuous performance task.

While one might argue that the findings of greater FMRI activation in women seen in our study might be the result of a baseline increase in regional glucose utilization and/or blood flow in women, one recent study refutes this notion [28]. A blood oxygenation level dependent FMRI study utilizing photic stimulation was performed in eight right-handed men and women of similar ages. Women exhibited a statistically significant decrease in the volume and degree of activation in the occipital lobes [28]. In another report by the same group, looking at a smaller sample, no gender effects were noted [32], though the amplitude of activation was diminished in elderly subjects. Therefore, in sensory stimulation functional imaging studies, one cannot conclude that women, by virtue of higher global rates of glucose metabolism, will always show 
greater FMRI activation than men. Each paradigm must be specifically tested.

\subsection{Individual vs. group maps}

We found interesting discrepancies between group-averaged maps and individual analyses. The group maps suggest that (1) by pooling data, activation that is occurring at low levels may reach significance in some areas while (2) areas of activation in single subjects may not be represented in group maps. As an example, though we identified entorhinal-piriform cortex activation in four men, the group maps showed no such activation. This may cause some difficulties when applying these data to subjects with abnormal senses of smell. When FMRI is applied to a test subject to assess for a 'normal response', one may be comparing that subject's individual analysis with that of the group norm. An area of activation on the test case may be present in many of the individual maps that make up the group norm yet not reach significance in the final group map. For these reasons, we believe it is important to report both individual and group-averaged activation results when performing FMRI studies such as these.

\subsection{Significance}

What is the value in studying cortical maps of olfaction? In addition to expanding the understanding of how the brain works and what circuits are integrated for a normal sense of smell, mapping of olfactory centers has important clinical implications. Patients suffering from dysosmia and parosmia are sometimes incapacitated by the disability associated with their chemosensory dysfunction. This can even affect the nutritional status of the individual; in some cases, an individual may fail to obtain the proper nutrition due to the unpleasant odors associated with food intake [13]. Knowing the potential sites for inducing changes in one's sense of smell prior to operating would be useful for surgeons who operate in the orbitofrontal regions (for craniofacial resections of head and neck cancers or skull base neoplasms like meningiomas) and periamygdaloid zones (for seizure focus or neoplasm resections). Specific deficits in olfaction may be shown to correlate with specific sites in the brain by FMRI and may help neurologists to better localize disease processes in advance of imaging.

There are also a whole host of diseases that can affect the sense of smell. Many of these diseases are neurodegenerative in nature and, since the sense of smell is affected in the aging process, FMRI data stratified by age will provide a framework upon which to study such entities as Alzheimer's disease, Parkinson's disease, Huntington's chorea, multiple sclerosis, Wernicke's encephalopathy, and Korsakoff's psychosis [10-13,29,31]. We have already demonstrated the marked diminution in brain activation under passive conditions of smell stimulation in subjects with Alzheimer's disease (Yousem, D.M., Geckle, R.J., Doty, R.L. Olfactory deficits in neurodegenerative disorders. RSNA 1995 Abstract \#271).

The present work may provide a new avenue for screening individuals at risk for developing Alzheimer's disease (e.g., with apolipoprotein-E karyotype E-4 or with early memory loss) or other neurodegenerative disorders that affect olfaction. Already psychophysical tests have been able to identify a patient population at risk for developing Alzheimer's disease [2]. We have also noted a near-perfect correlation between the number of multiple sclerosis plaques in presumed 'olfactory eloquent' regions and deficits on odor identification tests [11]. As multiple sclerosis is the most common neurological disease in the young (along with schizophrenia and head trauma which we intend to study) and Alzheimer's disease is one of the most common in the elderly, we believe that pursuing imaging tests of olfaction is critical to understanding neuropsychological pathogenesis.

Olfactory stimulated FMRI may be able to detect brain deficits in patients with many of these neurological diseases at an earlier stage where pharmacological intervention may be of benefit.

\subsection{Conclusion}

Gender may have an effect on the results of FMRI studies employing olfactory stimulants. This effect should be considered when designing and interpreting OSFMRI studies in normal subjects and in patients with diseases that affect olfaction.

\section{Acknowledgements}

We recognize the early contributions of Dana Suskind in advancing this research effort. Alexandra Genow and Julia Berg also lent their assistance during the performance of these studies. This paper is supported by research grant\# PO1 DC 00161-15 from the National Institute on Deafness and Other Communication Disorders, National Institutes of Health.

\section{References}

[1] P.J. Andreason, A.J. Zametkin, A.C. Guo, P. Baldwin, R.M. Cohen, Gender-related differences in regional cerebral glucose metabolism in normal volunteers, Psychiatry Research 51 (1994) 175-183.

[2] A.W. Bacon, V. Hartwell, R. Quinones, M. Giancola, D.P. Salmon, M.W. Bondi, C. Murphy, The role of apolipoprotein-E and olfactory functioning in people with Alzheimer's disease, International Symposium on Smell and Taste, San Diego, 1997.

[3] L.R. Baxter Jr., J.C. Mazziotta, M.E. Phelps, C.E. Selin, B.H. Guze, L. Fairbanks, Cerebral glucose metabolic rates in normal human females versus normal males, Psychiatry Research 21 (1987) 237245.

[4] G.G. Briggs, R.D. Nebes, Patterns of hand preference in a student population, Cortex 11 (1975) 230-238. 
[5] D.A. Deems, R.L. Doty, Age-related changes in the phenyl ethyl alcohol odor detection threshold, Transactions-Pennsylvania Academy of Ophthalmology and Otolaryngology 39 (1987) 646-650.

[6] D.A. Deems, R.L. Doty, R.G. Settle, V. Moore-Gillon, P. Shaman, A.F. Mester, C.P. Kimmelman, V.J. Brightman, J.B. Snow Jr., Smell and taste disorders, a study of 750 patients from the University of Pennsylvania Smell and Taste Center, Archives of Otolaryngology — Head and Neck Surgery 117 (1991) 519-528.

[7] R.L. Doty, Influence of age and age-related diseases on olfactory function, Annals of the New York Academy of Sciences 561 (1989) 76-86.

[8] R.L. Doty, S. Applebaum, H. Zusho, R.G. Settle, Sex differences in odor identification ability: a cross-cultural analysis, Neuropsychologia 23 (1985) 667-672.

[9] R.L. Doty, W.E. Brugger, P.C. Jurs, M.A. Orndorff, P.J. Snyder, L.D. Lowry, Intranasal trigeminal stimulation from odorous volatiles: psychometric responses from anosmic and normal humans, Physiology and Behavior 20 (1978) 175-185.

[10] R.L. Doty, D.A. Deems, S. Stellar, Olfactory dysfunction in parkinsonism: a general deficit unrelated to neurologic signs, disease stage, or disease duration, Neurology 38 (1988) 1237-1244.

[11] R.L. Doty, C. Li, L.J. Mannon, D.M. Yousem, Olfactory dysfunction in multiple sclerosis [letter], New England Journal of Medicine 336 (1997) 1918-1919.

[12] R.L. Doty, D.P. Perl, J.C. Steele, K.M. Chen, J.D. Pierce Jr., P. Reyes, L.T. Kurland, Olfactory dysfunction in three neurodegenerative diseases, Geriatrics 46 (1991) 47-51.

[13] R.L. Doty, P. Shaman, S.L. Applebaum, R. Giberson, L. Siksorski, L. Rosenberg, Smell identification ability: changes with age, Science 226 (1984) 1441-1443.

[14] W.J. Evans, L. Cui, A. Starr, Olfactory event-related potentials in normal human subjects: effects of age and gender, Electroencephalography and Clinical Neurophysiology 95 (1995) 293-301.

[15] K. Friston, J. Ahburner, J. Poline, C. Frith, J. Heather, R. Frackowiak, Spatial realignment and normalization of images, Human Brain Mapping 2 (1995) 202-214.

[16] K. Friston, A. Holmes, K. Worsley, J. Poline, C. Frith, R. Frackowiak, Statistical parametric maps in functional imaging: a general approach, Human Brain Mapping 2 (1995) 189-201.

[17] K.J. Friston, Commentary and opinion: II. Statistical parametric mapping: ontology and current issues, Journal of Cerebral Blood Flow and Metabolism 15 (1995) 361-370.

[18] K.J. Friston, C.D. Frith, P.F. Liddle, R.J. Dolan, A.A. Lammertsma, R.S. Frackowiak, The relationship between global and local changes in PET scans, Journal of Cerebral Blood Flow and Metabolism 10 (1990) 458-466, [see comments].

[19] K.J. Friston, C.D. Frith, P.F. Liddle, R.S. Frackowiak, Comparing functional (PET) images: the assessment of significant change, Journal of Cerebral Blood Flow and Metabolism 11 (1991) 690-699.

[20] K.J. Friston, K.J. Worsley, R.S.J. Frackowiak, J.C. Mazziotta, A.C. Evans, Assessing the significance of focal activations using their spacial extent, Human Brain Mapping 1 (1994) 214-220.

[21] A.N. Gilbert, M.S. Greenberg, G.K. Beauchamp, Sex, handedness and side of nose modulate human odor perception, Neuropsychologia 27 (1989) 505-511, [published erratum appears in Neuropsychologia 1989; 27 (10), p. 1313].

[22] R.C. Gur, L.H. Mozley, P.D. Mozley, S.M. Resnick, J.S. Karp, A. Alavi, S.E. Arnold, R.E. Gur, Sex differences in regional cerebral glucose metabolism during a resting state, Science 267 (1995) $528-531$.

[23] R.E. Gur, R.C. Gur, Gender differences in regional cerebral blood flow, Schizophrenia Bulletin 16 (1990) 247-254.

[24] J. Hatazawa, R.A. Brooks, G. Di Chiro, G. Campbell, Global cerebral glucose utilization is independent of brain size: a PET Study, Journal of Computer Assisted Tomography 11 (1987) 571576.

[25] T. Hummel, H. Pietsch, G. Kobal, Kallmann's syndrome and chemosensory evoked potentials, European Archives of Oto-RhinoLaryngology 248 (1991) 311-312.

[26] G. Kobal, C. Hummel, Cerebral chemosensory evoked potentials elicited by chemical stimulation of the human olfactory and respiratory nasal mucosa, Electroencephalography and Clinical Neurophysiology 71 (1988) 241-250.

[27] I. Koizuka, H. Yano, M. Nagahara, R. Mochizuki, R. Seo, K. Shimada, T. Kubo, T. Nogawa, Functional imaging of the human olfactory cortex by magnetic resonance imaging, Journal of OtoRhino-Laryngology and its Related Specialties 56 (1994) 273-275.

[28] J.M. Levin, M.H. Ross, J.H. Mendelson, N.K. Mello, B.M. Cohen, P.F. Renshaw, Sex differences in blood-oxygenation-level-dependent functional MRI with primary visual stimulation, American Journal of Psychiatry 155 (1998) 434-436.

[29] C. Li, D.M. Yousem, R.L. Doty, D.W. Kennedy, Neuroimaging in patients with olfactory dysfunction, American Journal of Roentgenology 162 (1994) 411-418.

[30] S.A. Miura, M.B. Schapiro, C.L. Grady, A. Kumar, J.A. Salerno, W.E. Kozachuk, E. Wagner, S.I. Rapoport, B. Horwitz, Effect of gender on glucose utilization rates in healthy humans: a positron emission tomography study, Journal of Neuroscience Research 27 (1990) 500-504.

[31] P.J. Moberg, G.D. Pearlson, L.J. Speedie, J.R. Lipsey, M.E. Strauss, S.E. Folstein, Olfactory recognition: differential impairments in early and late Huntington's and Alzheimer's diseases, Journal of Clinical and Experimental Neuropsychology 9 (1987) 650-664.

[32] M.H. Ross, D.A. Yurgelun-Todd, P.F. Renshaw, L.C. Maas, J.H. Mendelson, N.K. Mello, B.M. Cohen, J.M. Levin, Age-related reduction in functional MRI response to photic stimulation, Neurology 48 (1997) 173-176.

[33] N. Sobel, V. Prabhakaran, J.E. Desmond, G.H. Glover, R.L. Goode, E.V. Sullivan, J.D.E. Gabrieli, Sniffing and smelling: separate subsystems in the human olfactory cortex, Nature 392 (1998) 282-285.

[34] Q.X. Yang, B.J. Dardzinski, S. Li, P.J. Eslinger, M.B. Smith, Multi-gradient echo with susceptibility inhomogeneity compensation (MGESIC): demonstration of fMRI in the olfactory cortex at $3.0 \mathrm{~T}$, Magnetic Resonance in Medicine 37 (1997) 331-335.

[35] D.M. Yousem, S.C. Williams, R.O. Howard, C. Andrew, A. Simmons, M. Allin, R.J. Geckle, D. Suskind, E.T. Bullmore, M.J. Brammer, R.L. Doty, Functional MR imaging during odor stimulation: preliminary data, Radiology 204 (1997) 833-838.

[36] D.H. Zald, J.V. Pardo, Emotion, olfaction, and the human amygdala: amygdala activation during aversive olfactory stimulation, Proceedings of the National Academy of Sciences of the United States of America 94 (1997) 4119-4124.

[37] R.J. Zatorre, M. Jones-Gotman, A.C. Evans, E. Meyer, Functional localization and lateralization of human olfactory cortex, Nature 360 (1992) 339-340. 